\title{
Wear progress of nitrided microstructures in tribological simulation of aluminium hot extrusion
}

\author{
M. Tercelj, I. Perus \& G. Kugler \\ Department of Materials and Metallurgy, Faculty of Natural Sciences \\ and Engineering, University of Ljubljana, Slovenia
}

\begin{abstract}
The study of wear of a tribological pair of soft and heated metals in contact with a hard counter body is very demanding. So far, less attention has been devoted to this problem and thus progress in this field is needed. In Al hot extrusion also the mentioned tribological system prevails but it is also supposed that various contact pressures between extruded $\mathrm{Al}$ die bearing surfaces takes place. In order to explain wear behaviour on die bearing surfaces wear tests of nitrided surfaces at low, medium and higher contact pressures using block on cylinder test equipment were carried out. Tested surfaces at medium contact pressures exhibit some significances obtained at testing at lower as well as at higher contact pressures. Essentially, the difference at medium contact pressure is higher density of partial removal of compound layer on spots of its previous initial cracking in comparison to lower contact pressure. At higher contact pressure removal of the compound layer is already preferentially oriented in the sliding direction in the first stage, while at medium contact pressure this is observed only in later stages of the degradation progress.
\end{abstract}

Keywords: aluminium hot extrusion, die wear progress, nitrided microstructures, compound layer, cracking.

\section{Introduction}

Hot extrusion is normally used for forming long Al products, like rods, beams, wires, etc. Over $95 \%$ of all extrusion dies are gas nitrided since application of PVD coatings has some limitations due to "line-of-sight" effect at coating in narrow gape of complex shaped dies. In order to increase the service time of the 
dies for aluminium hot extrusion it is essential to find the degradation paths of the nitrided surface layer on the bearing surface. In Al hot extrusions various damages on nitrided bearing surfaces of dies can be observed and the reasons for their occurrence and their different previous progress are still not sufficiently experimentally clarified [1-4]. During nitriding the die steels, in general two different types of microstructure are formed on the die surface, i.e. microstructure with "compound layer" on top and diffusion layer under it, and in the second case, diffusion layer alone. Compound layer usually consists of brittle iron nitrides, i.e. $\varepsilon$ phase $(\varepsilon-\mathrm{Fe} 2-3 \mathrm{~N}), \gamma^{\prime}$ phase $\left(\gamma^{\prime}-\mathrm{Fe} 4 \mathrm{~N}\right)$ or mixed phases $\left(\varepsilon+\gamma^{\prime}\right)$. When slightly higher contact pressures (about $20 \mathrm{MPa}$ ) are applied, the compound layer usually spalls off the die surface at the very beginning of the forming process, and this usually essentially accelerates the process of wear. Microstructure after nitriding as well as die design should be in each application adapted to actual loads that prevail on the die. It well known that nitrided microstructure of dies aimed for Al hot extrusion is very important at prolongation of die life [5-10]. Study of wear of tribological pair of soft and heated metal in contact with hard counter body is very demanding. So far less attention was devoted to this problem and thus progress on this field is needed. The main difficulty of such studies is deficiency of reliable experimental data due to the problem of maintenance of prescribed contact pressures for longer times between softer metals, which are heated close to melting temperature, and hard body. Although some laboratory investigations have partially revealed the complexity of the wear process, the progress of wear in a tribological system composed of soft and hot $\mathrm{Al}$ in a sliding contact with a hard specimen and, consequently, the various die life times and the damage to the bearing surfaces of the dies are still not well understood. The main reasons for this are the difficulties in establishing and maintaining the appropriate tribological system for laboratory testing that corresponds to Al hot extrusion, where the soft and hot $\mathrm{Al}$ is in a sliding contact with a hard specimen. In order to be able to study the wear progress in $\mathrm{Al}$ hot extrusion it is essential to conduct the experiments at a variety of contact pressures [1-4]. Therefore, the aim of the present contribution is to employ appropriate testing conditions to study the degradation of a compound layer of nitrided microstructures at various contact pressures.

\section{Test equipment, test conditions, applied methods and materials}

In order to study wear mechanisms at medium and higher contact pressures wear testing has been conducted using six blocks. Nitrided blocks for testing were made of AISI H13 and of H10 tool steels, with various characteristics of compound as well as of diffusion layers.

Block on cylinder equipment was used for testing of wear (see Figure 1). With different ratio of heat supply on Al cylinder, i.e. from outside (direct heating via heating coil) and from inside (indirect heating via steel discs), at approximately the same radial outer surface temperature of $\mathrm{Al}$ cylinder in radial direction, different temperature gradients and consequently also different contact 


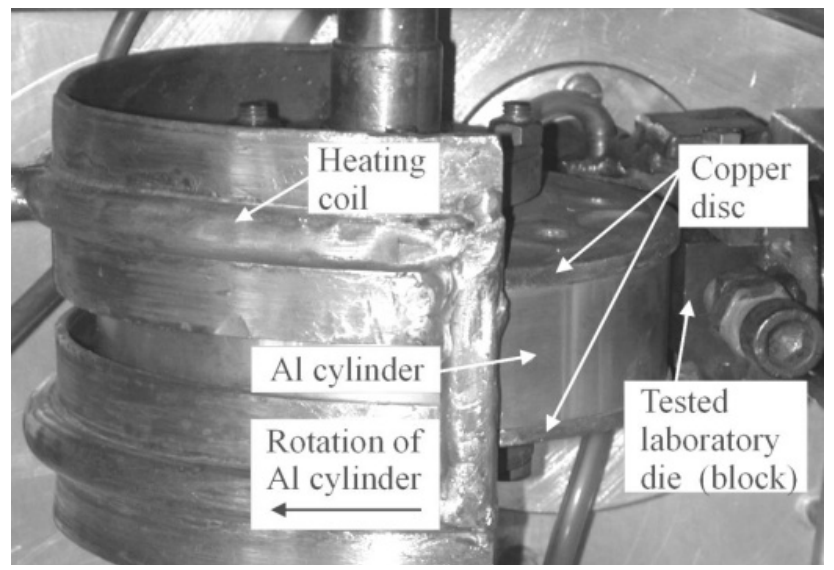

Figure 1: Block on cylinder testing equipment.

lengths between $\mathrm{Al}$ cylinder and tested tool steel block at the same normal force of $1920 \mathrm{~N}$ were established. As a consequence also various contact pressures can be applied for wear testing. In our case for medium contact pressures the contact length of around $10 \mathrm{~mm}$ and for higher contact pressures of around $7 \mathrm{~mm}$ were established and applied in testing. Contact lengths that have been established at testing for each used block are collected in Table 1. Note that contact length of about $13 \mathrm{~mm}$ was considered as lower contact pressure. Temperature of $\mathrm{Al}$ cylinder was assumed to be about $550{ }^{\circ} \mathrm{C}$ but outer temperature of sliding surface is assessed to be slightly below $600{ }^{\circ} \mathrm{C}$, and relative sliding velocity of $\mathrm{Al}$ cylinder was $0.5 \mathrm{~m} / \mathrm{s}$. In order to gain more information about degradation process on tested surface the results after 30,60, $120 \mathrm{~min}$ of testing are given.

Table 1: Chemical composition of the die steels used.

\begin{tabular}{|c|c|c|c|c|c|c|c|c|}
\hline AISI & Block & $\mathbf{C}$ & Si & Mn & $\mathbf{C r}$ & Mo & $\mathbf{V}$ & Fe \\
\hline H13 & $2,4,6$ & 0.40 & 1.0 & 0.40 & 5.05 & 1.28 & 0.95 & rest \\
\hline H10 & $1,3,5$ & 0.30 & 0.30 & 0.38 & 3.0 & 2.80 & 0.45 & rest \\
\hline
\end{tabular}

To obtain microhardness, microstructure and phases of nitrided surfaces the following equipment was used: microhardness by Fischerscope H100C, microstructures by OLYMPUS BX60M optical microscope and X-ray diffraction (XRD) investigations of compound layer by Siemens Analytical D5000 diffractometer with $\mathrm{Cu}$ anode. On tested surface also energy dispersive spectroscopy, scanning electron microscope, and back-scattered electrons analyses were carried out using JEOL JSM 5610 equipment. Microstructures have been prepared using nital etchant. 


\section{Results and discussion}

\subsection{Initial microstructures and XRD of samples and applied industrial die}

The obtained microstructures of the nitrided blocks are shown in Figure 2, while their main characteristics are given in Table 2. The thickness of the compound layer on blocks $2,4,6$ is about $8-10 \mu \mathrm{m}$, with a nitriding depth of $110 \mu \mathrm{m}$, while the maximum microhardness amounts to $1130 \mathrm{Hv}$. No nitrides were observed on the grain boundaries of this block. The nitriding depth of block 2, 4 and 6 is approximately the same as for block 1,3 and 5 but the compound layer is thicker, i.e., $9-11 \mu \mathrm{m}$, and there are nitrides present on the grain boundaries.

a)

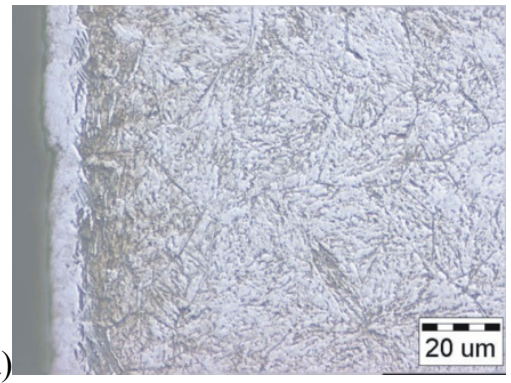

b)

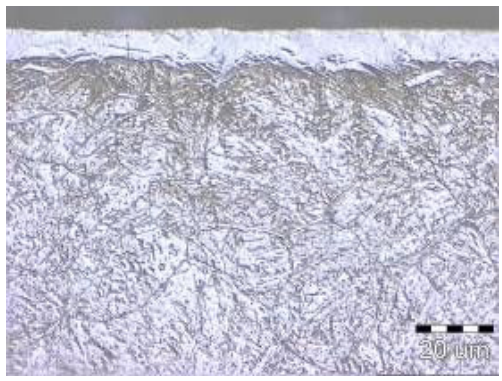

Figure 2: $\quad$ Initial microstructures of the tested blocks: block 1 (a), block 2 (b).

Table 2: $\quad$ Main values of nitrided blocks ${ }^{1}$.

\begin{tabular}{|c|c|c|}
\hline & Blocks 1,3,5 & Blocks 2, 4, 6 \\
\hline MMH $[\mathrm{Hv}]$ & 1100 & 1130 \\
\hline ND $[\mu \mathrm{m}]$ & 110 & 110 \\
\hline CLT $[\mu \mathrm{m}]$ & $9-11$ & $8-10$ \\
\hline
\end{tabular}

${ }^{1} \mathrm{MMH}-$ maximal microhardness, ND-nitriding depth,

CLT-compound layer thickness.

The XRD patterns of the nitrided surfaces revealed that the compound layers on blocks are almost $\varepsilon$-mono-phased, since the amount of $\gamma^{\prime}$ phase $\left(\mathrm{Fe}_{4} \mathrm{~N}\right)$ is very low. Note that the absence of porosity results in decreased brittleness of the compound layer [5-10]. Thus these characteristics of nitrided blocks do not differ essentially to each other but at compound layer removal different density of porosity was found at both groups of blocks that resulted in different wear rates.

\subsection{Wear progress on nitrided blocks at lower contact pressure}

The main characteristics that can be observed after 30 min of testing of block 1 are two initial types of degradation. The first one is based on initial cracking and the second one is based on initial partial fragmental removal of the compound 
layer without cracking (see Figure 3a). The first type of degradation is predominately related to the occurrence of porosity in the compound layer as is presented in detail for the next stage of the degradation progress in Figure 3b. On the other hand, from Figure 3a we can see the fragmental removal of the compound layer without any observed porosity in the compound layer on spots of its removal and small cracks with a low depth together with the removed compound layer around these cracks. As mentioned the next step of the degradation process on the tested surface, which corresponds to further degradation of the compound layer, are the spots of partial removal of the compound layer, denoted in Figure $3 b$.

a)

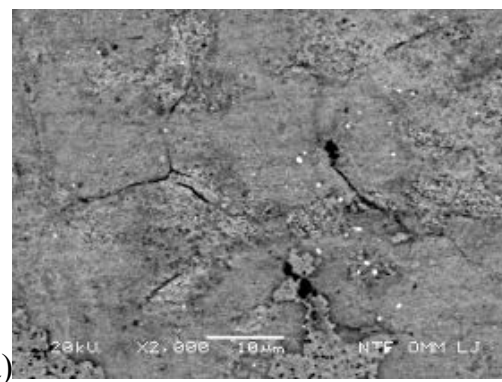

c

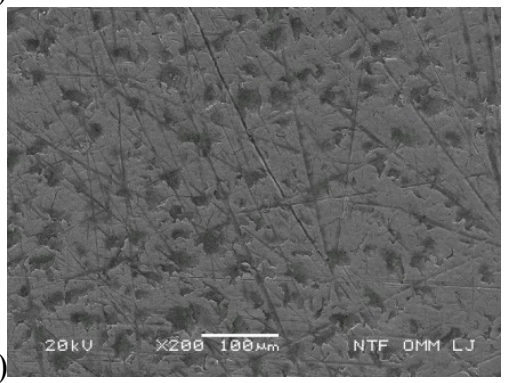

e)

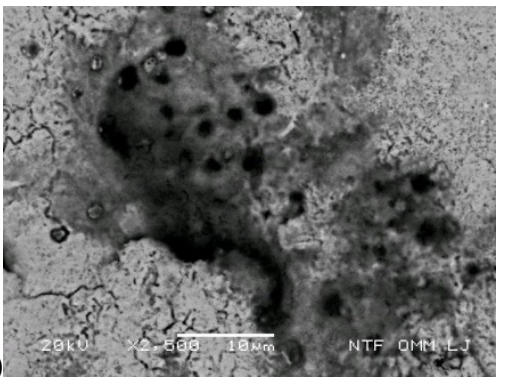

b)

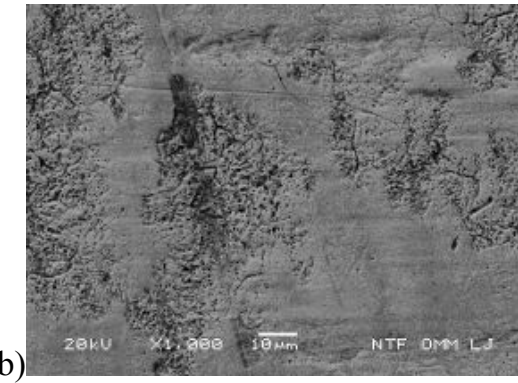

d)

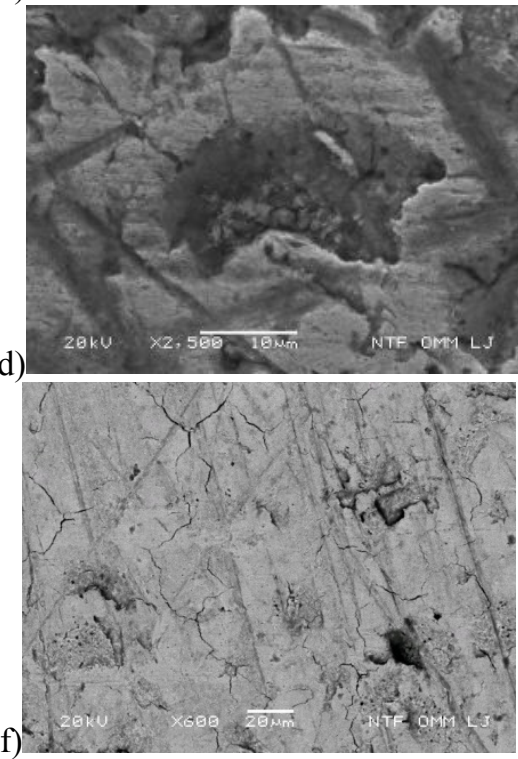

Figure 3: Main wear characteristic of surface of block 1 after 30 min of testing; initial surface micro-cracking (a), removal of compound layer around initial cracks (b) and after 60 min of testing, main damage characteristic (c), with detail of islet of complete removal of the compound layer (d), tear off of the compound layer (e), cracking of compound layer (f), sliding direction $\leftarrow$. 
These figures show that the initial cracking behaviour can be ascribed to the presence of porosity. The second type of fragmental removal without any emphasized initial surface cracking of the compound layer shown in Figure 3a can be ascribed to the adhesion between the hot $\mathrm{Al}$ and the compound layer. Initially the surface roughness is relatively low in comparison to the roughness of the areas where partial removals have already taken place (see Figure 3a). A lower roughness leads to a lower friction and consequently the amount of fragments removed due to adhesion is decreased in these areas.

From these figures it can be concluded that the rate of removal of the compound layer at the observed spots is low, revealing a stepwise process of partially removing thin and small fragments. After 60 min of testing of block 1 the degradation process continues and results in the increased cracking of the compound layer, as shown in Figure 3c. Also an increased number of areas with a partial removal of the compound layer (similar to Figure $3 a$ ) can be seen, as well as a larger number of small islands where the compound layer was removed up to the diffusion layer (see detail of the complete removal in Figure 3d). As expected, an increased number of spots where the compound layer was only partly removed with porosity on the spots of the removal can also be seen (see Figures 3e-f). It was found that three different types of such removal can be distinguished. The first type of removal, named crack spreading of the compound layer, begins on spots where the pores are close to the surface. This type of removal is a consequence of the normal pressure and friction, and probably appeared just before the interruption of the 60-min testing. Another type of compound-layer removal can be observed on places where the porosity is present and the appearance of a new surface (see Figure 3f) indicates that the stepwise removal of the compound layer is a consequence of the adhesion between the hot $\mathrm{Al}$ and the compound layer.

The third type of intensive compound-layer removal occurs at spots of triplepoint cracking, as shown in Figure 3f. These spots are more brittle and have a lower strength due to the porosity that is usually present there. A comparison of the surfaces between samples after 30 and $60 \mathrm{~min}$ of testing revealed an increased number of cracks in the compound layer for the latter, which contributes to the degradation in the next stage of the wear process. For the same testing times the tribologically loaded surface on block 2 suffered considerably less damages than block 1 . Thus only small fragmental removal of the compound layer based on adhesion as well as on spalling, as a consequence of previous surface cracking, was observed on the surface of block 2 after 30 min of testing, which indicates the better quality of the compound layer of block 2 in comparison to block 1 .

After two hours of testing block 1, the following observations were made (Figure 4): (i) continuation of the cracking of the compound layer in terms of width and depth, (ii) the appearance of new cracks on the surface, (iii) the process of partial removal of the compound layers due to the presence of porosity, (iv) cracking and fragmental removal of the diffusion layer, and (v) there are mainly areas with partial removal and areas of complete removal of the compound layer where diffusion-layer cracking and the fragmental removal 
can be observed; thus there are almost no undamaged areas on the tested surface. These observations will be explained in more detail below. Figure $4 \mathrm{~b}$ shows details of Figure $4 \mathrm{a}$, where it can be observed that the process of removal progressed to such an extent that the beginning of the fragmental removal (spalling) of the diffusion layer is visible. Besides the cracking of the diffusion layer, which has a large influence on the wear, a chemical attack can also be observed on these figures. The cracking can be attributed to the increased friction between the tested surface and the Al cylinder. Namely, hot aluminium in contact with the diffusion layer has a tendency to stick to it, but due to the rotation of the $\mathrm{Al}$ cylinder this sticking is interrupted. Repetition of this process leads to the surface cracking of the diffusion layer which can be regarded as the first stage of diffusion-layer degradation and its fragmental spalling (fragmental tear off) as the next stage. The fragmental spalling of the diffusion layer is presented in Figure 4c, where spalling and to a lesser extent also chemical attack are clearly visible. From these results it can be concluded that the dominant wear mechanism is spalling and not chemical attack, which is in contrast to the prevailing opinion in the scientific literature [1--4], where chemical attack is regarded as the main wear mechanism. From Figure $4 \mathrm{~d}$ is it can be concluded that the porosity is responsible for the initial micro-cracking and its expansion.

a)
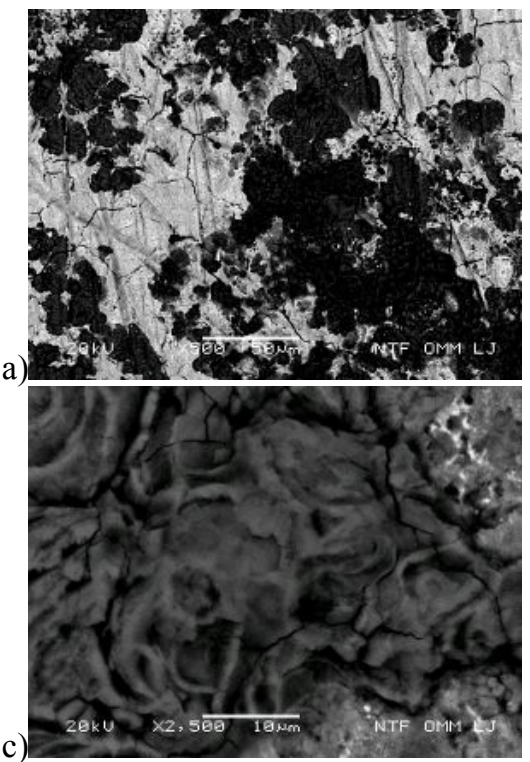
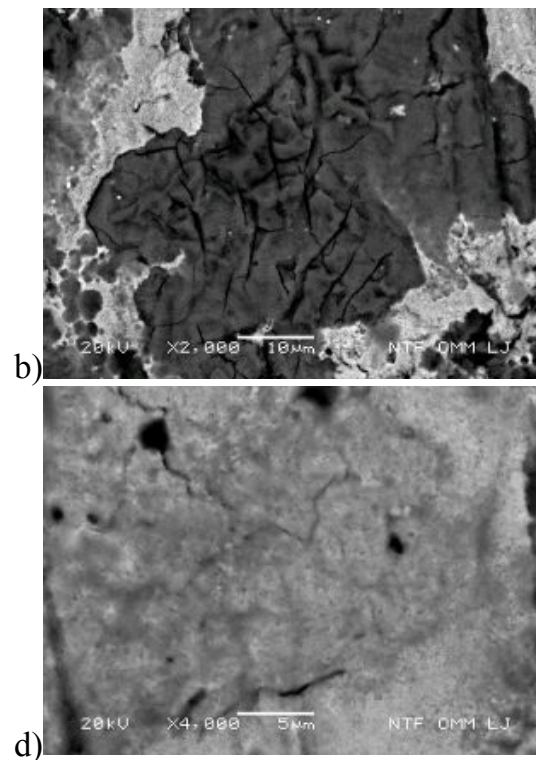

Figure 4: Main wear characteristic of block 1 after $120 \mathrm{~min}$ of testing, appearance of the tested surface (a), detail of fragmental removal of less damaged surface (b), detail of fragmental removal with chemical attack of more damaged surface (c), detail of initial cracking at porosity (d), sliding direction $\leftarrow$. 
Images of block 2 after one hour of testing are presented in Figure 5a. It can be seen that there are fewer spots where the compound layer was removed down to the diffusion layer and less cracking in comparison to block 1 for the same time of testing. Spots with the partially removed fragments as shown in Figure $5 \mathrm{~b}$ revealed that the porosity in the compound layer is responsible for its degradation, since on the removed surfaces of block 2. Note that from the scientific literature [5-10] it is well known that porosity is a consequence of inappropriate nitriding parameters. Furthermore occurrence of the surface microand macro-cracking as well as the spots where comparatively small fragments were removed was observed. These phenomena are usually a consequence of the normal contact pressure and the strong adhesion between the $\mathrm{Al}$ and the nitrided surface as well as of the presence of porosity in the compound layer. However, also spots with the partial removal of the compound layer as a consequence of the normal contact pressure without any visible porosity on the removed were observed. Thus, it can be concluded that further compound-layer removal also occurs due to its micro-cracking and the small fragmental removal as a consequence of the adhesion.

a)
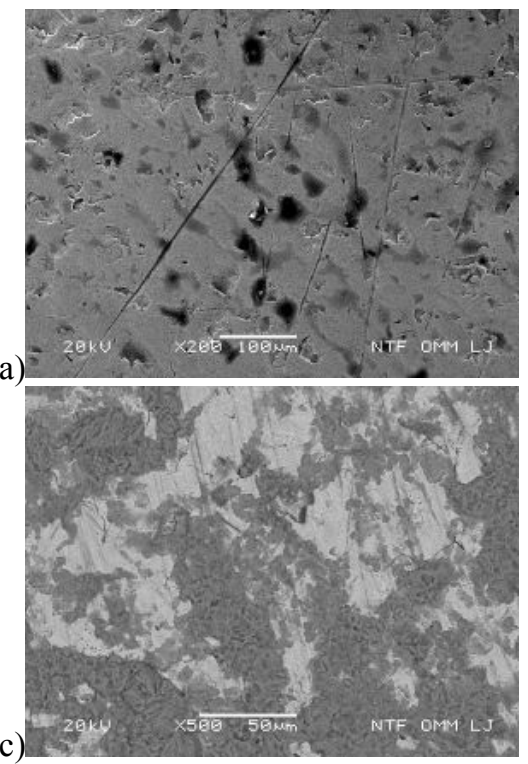

b)

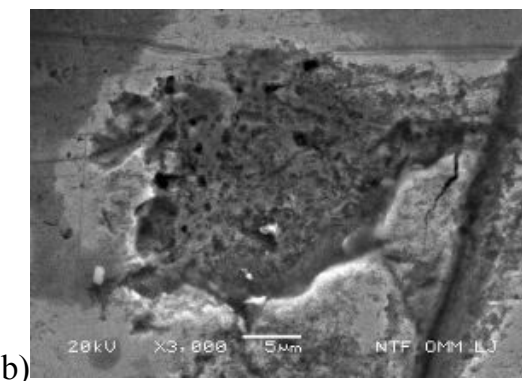

d)

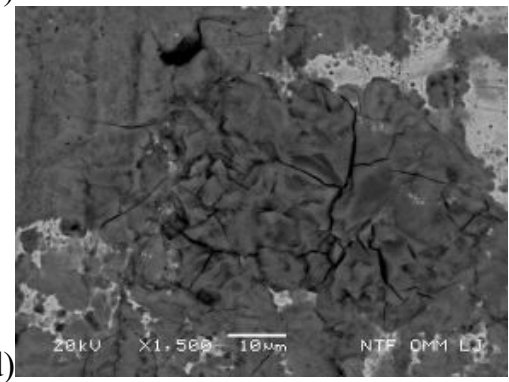

Figure 5: Main wear characteristic of tested surface of block 2 after: 60 min of testing (a) with detail of partial removal of compound layer (b), and after 120 min testing: removal of the compound layer, beginning of the fragmental removal of the compound layer (c), detail of beginning of fragmental removal (d) sliding direction $\leftarrow$.

After two hours of testing of block 2 the areas where the compound layer is completely removed are larger (see Figure 5c). In a detailed view of this figure, as shown in Figure 5d, increased roughness as a consequence of surface cracking 
and partial removal is visible on the top of the diffusion layer. It is also clear that the density of the cracks, both on the partially removed compound layer and on the diffusion layer, is considerably lower than for block 1 .

\subsection{Wear progress of nitrided surfaces at medium and high contact pressures}

Figure 6a shows the appearance of the tribologically loaded surface of block 4 after 30 minutes of testing, where the partial removal in the shape of small islets, i.e., micro-craters with diameters of about $20-30 \mu \mathrm{m}$, can be distinguished. A detail of this type of removal is shown in Figure $6 \mathrm{~b}$, where on spots of the compound layer's partial removal, a porosity of lower density can be observed. It can be assumed that this type of compound-layer degradation is based on two different mechanisms: (i) on strong adhesion, and (ii) initial surface cracking of the compound layer due to the presence of porosity.

The first assumption is based on the observation of smaller removed islets where no cracking was found around these spots. Thus, the process of degradation, which is based on adhesion around these islets, results in the partial removal of the compound layer. The presence of the porosity does not always result in initial cracking, but sometimes in an initial removal by adhesion. In the latter case, the presence of porosity in the compound layer accelerates its removal and is a consequence of the decreased shear strength at such spots, and thus thicker and larger areas of the compound layer can be removed. On the other hand, the combination of the presence of porosity and a sufficiently high contact pressure results in the second mechanism, i.e., the initial surface cracking of the compound layer, which is then followed by adhesional removal.

After $60 \mathrm{~min}$ of testing of block 4 the density of micro-craters is increased as well as their width and depth (see Figure 6c). It is clear that the islets of the initially partly removed compound layer increased in depth and width and the diameters of the micro-craters are about $25-45 \mu \mathrm{m}$. Additionally, the coalescence of some islets has occurred, as shown in Figure 6c. Small islets where the compound layer was completely removed up to the diffusion layer as well as some larger areas where the compound layer was removed preferentially in the sliding direction of the Al cylinder (see Figure 6d), can also be distinguished. The latter can be attributed to the locally poorer characteristics of the compound layer, i.e., the local thickness of the compound layer and/or the porosity are increased. This, in combination with the increased adhesion and contact pressure, results in larger removed areas, which are preferentially oriented in the sliding direction. On the other hand, the occurrence of small islets with a deeper removal (Figure 6c) can be attributed to the locally emphasized increased density of the porosity through the entire thickness of the compound layer, which consequently leads to an accelerated adhesional removal at such spots. Thus it is reasonable to conclude that adhesion as a consequence of the high contact pressure is responsible for the accelerated compound-layer removal. 
a)
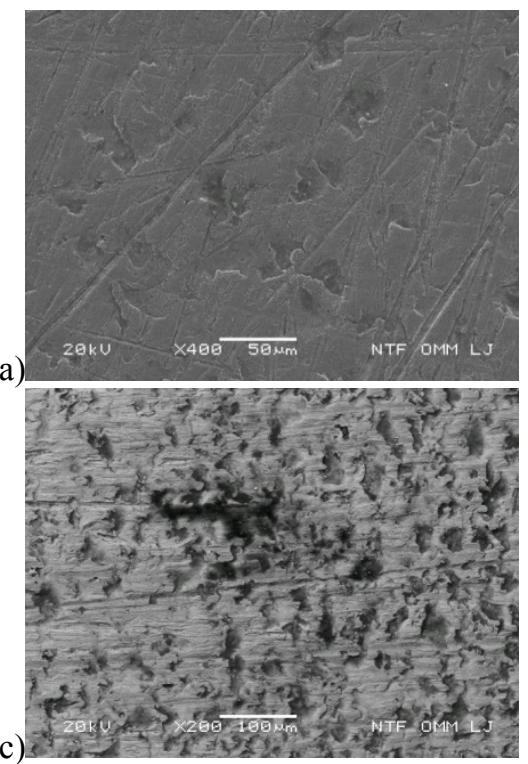

b)

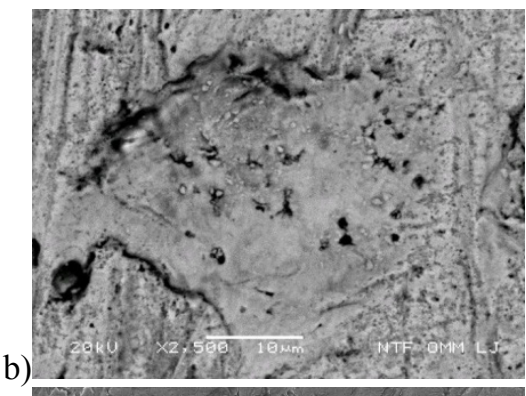

d)

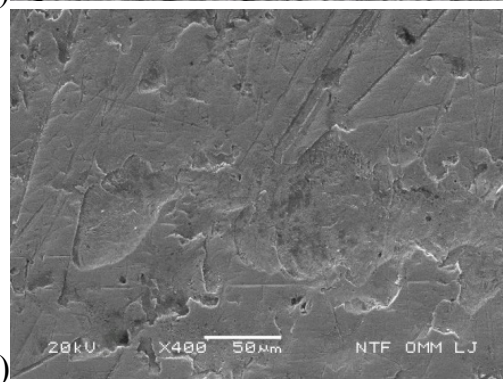

Figure 6: $\quad$ Tested surface of block 1 after 30 min testing: appearance of microcraters on the tested surface (a) with detail of the micro-craters with observed porosity (b), and after 60 min: micro-craters on the tested surface (c), areas with the removal of the compound layer preferentially in the sliding direction (d), sliding direction $\leftarrow$.

After 120 min of testing of block 4 an increased density of deeper islets, where the removal exceeds the compound-layer thickness, can be observed on the nitrided surface (see Figure 7a). The depths that extend into the diffusion layer, the widths and the density of the micro-craters are considerably increased for this surface in comparison to that observed after 60 min of testing. In Figure $7 \mathrm{a}$ another phenomenon that is related to the increased friction is also visible, i.e., the unification of the micro-craters. Those with thinner walls and a greater depth tend to lie perpendicular to the sliding direction. An accelerated degradation of the diffusion layer can be expected on these spots. Of course on block 3, with worse characteristics of compound layer in comparison to block 4, the process of formation of micro-craters was faster thus after two hours of testing compound layer was completely removed as presented on Figure $7 \mathrm{~b}$ since above mentioned unification of the micro-craters occurred on entire tested surface.

Blocks 5 and 6 were applied for testing at high contact pressures. At both blocks after 30 min of testing removal of compound layer was preferentially oriented in sliding direction and wear rate was increased in comparison to testing at medium contact pressure. Already after $60 \mathrm{~min}$ of testing compound layer was almost completely removed. 
a)

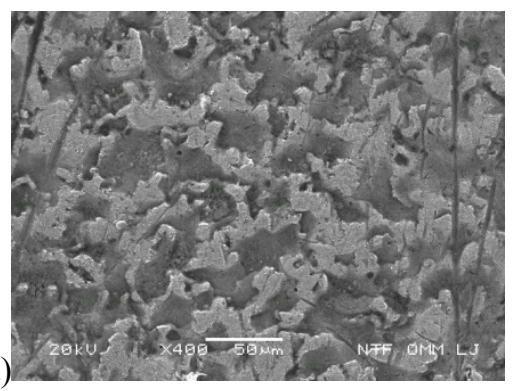

b)

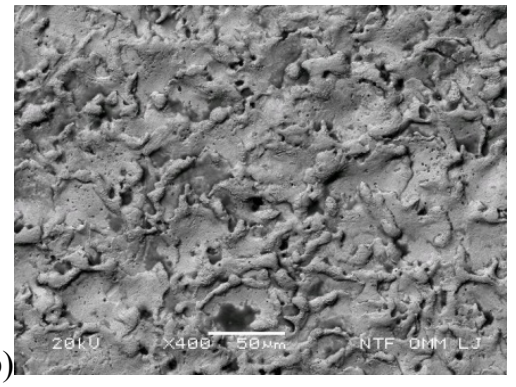

Figure 7: $\quad$ Main wear characteristic of surface of blocks 3 and 4 after $120 \mathrm{~min}$ of testing: the occurrence of denser micro-craters due to strong adhesion (a) removed compound layer (b); sliding direction $\leftarrow$.

\section{Conclusions}

The tribological system present in Al hot extrusion has been simulated using block-on-cylinder wear testing equipment. The degradation progress of nitrided surfaces has been investigated at low, medium and high contact pressures. The following conclusions can be drawn:

- Nitrided surfaces differ in density of porosity that results in different wear rate of nitrided surfaces. The contact pressure and characteristics of the nitrided microstructure have a decisive role in determining wear mode. For all the applied contact pressures, the characteristics of the compound layers influence the time of the occurrence of a specific degradation mode.

- For all the tested contact pressures, adhesion is the main wear mechanism. The surfaces tested at a medium contact pressure have features that are characteristic for low as well as for high contact pressures, respectively, i.e., the formation of micro-craters is mostly encountered at low contact pressures, while the removal of the compound layer that is oriented in the sliding direction is more emphasized at a high contact pressure. The formation of islets at a medium contact pressure starts with either a strong adhesional removal without cracking or with the surface cracking of the compound layer.

- The main difference between the results obtained at low and medium contact pressures is in the density of the removed islets and in their surface area at the same testing times. The next difference is that the extension of craters in the sliding direction does not appear at a low contact pressure.

- In the early stage of the compound-layer degradation at medium contact pressures the removal is not preferentially oriented, but it becomes so in the later stages of testing.

- At high contact pressures the removal of the compound layer is preferentially oriented in the sliding directions. 


\section{References}

[1] Björk T, Bergström J, Hogmark S. Tribological simulation of aluminium hot extrusion. Wear 1999;224:216-25. A.F.M. Arif, Effect of extrusion die profile on the uniformity of nitrided layer, Materials and Manufacturing Processes, 24 (2009) 619-625.

[2] T. Björk, R. Westegård, S. Hogmark, Wear of surface treated dies for aluminium extrusion- a case study, Wear, 249/2 (2001) 316-323.

[3] M. Tercelj, A. Smolej, T. Vecko-Pirtovsek and R. Turk, The Microstructures and Wear Resistance of Gas-Nitrided and Ionically Nitrided AISI H10 Dies with Narrow Gaps Designed for the Hot Extrusion of Aluminium, JSME Inter. J. Series C, 49 (2006) 121-128.

[4] Björk T, Bergström J, Hogmark S. Optimitization of nitriding parameters for extrusion dies, evaluated in an extrusion simulation, In: Seventh international aluminium extrusion technology seminar, 2 (2000) 317-25.

[5] S. S. Akhtar \& A. F. M. Arif \& Bekir Sami Yilbas, Evaluation of gas nitriding process with in-process variation of nitriding potential for AISI H13 tool steel, Int J Adv Manuf Technol 47 (2010) 687-698,

[6] G. Castro, A. Fernández-Vicente and J. Cid, Influence of the nitriding time in the wear behaviour of an AISI H13 steel during a crankshaft forging process, Wear 263 (2007) 1375-1385.

[7] M. Hernandez, M.H. Staia, E.S. Puchi-Cabrera, Evaluation of microstructure and mechanical properties of nitrided steels, Surface \& Coatings Technology 202 (2008) 1935-1943.

[8] J. Baranowska, K. Szczecinski, M. Wysiecki, Increasing of gas nitriding kinetics via surface pre-treatment, Surface and Coatings Technology 151 152 (2002) 534-539.

[9] D. Lidtke, U. Baudis, J. Bosslet, U. Huchel, H. Klümper-Westkamp, W. Leche, H. J. Spies, Warmebehandlung von Werkstoffen, Nitrieren und nitrocarburieren, ISBN 3-8169-2416-6, Expert Verlag, 2006.

[10] N. Krishnaraj, P. Bala Srinivasan, L.J.L. Iyer, S. Sundaresan, Optimitization of compound layer thickness for wear resistance of nitrocarburized H1 1 steel, Wear 215 (1998) 123-30. 